\title{
Six Sigma: Decreasing neonatal intraventricular hemorrhage by delayed umbilical cord clamping
}

\author{
Samantha Clark ${ }^{1}$, Abdelhamid Bourbia ${ }^{2}$, Nancy Marcus ${ }^{3}$, Dara Seybold ${ }^{4}$, Michelle Mullens ${ }^{5}$, Morgan Lough ${ }^{6}$ and Byron C Calhoun ${ }^{*}$ \\ ${ }^{1}$ Department of Obstetrics and Gynecology, West Virginia University-Charleston, 800 Pennsylvania Ave, Charleston, WV 25302 \\ ${ }^{2}$ Department of Pediatrics, Pediatrix, 800 Pennsylvania Ave, Charleston, WV 25302, USA \\ ${ }^{3}$ Neonatal Intensive Care Unit, Charleston Area Medical Center, Charleston, WV 25302, USA \\ ${ }^{4}$ Center for Health Services and Outcomes Research, Charleston Area Medical Center Health Education and Research Institute, USA \\ ${ }^{5}$ Labor and Delivery, Charleston Area Medical Center, Charleston, WV 25302, USA \\ ${ }^{6}$ West Virginia University-Charleston, Charleston, WV 25302, USA
}

\begin{abstract}
Aims: The objective of this study was the application of Six-Sigma methodology, specifically, the Define-Measure-Analyze-Improve-Control (DMAIC) and Lean tool to decrease the incidence if intraventricular hemorrhage (IVH) in preterm neonates $\leq 32$ weeks.

Methods: This was an observational study of changes resulting from the application of Six Sigma principles to prevention of intraventricular hemorrhage in preterm neonates $\leq 32$ weeks.

Using cohort analysis and Six Sigma principles, we compared the baseline process from 1 January 2014-31 December 2014 with our implemented intervention from 1 January 2015-31 December 2015.

Results: Using Lean and DMAIC tools (including “voice of the customer") and team-focused approach; intraventricular hemorrhage decreased from 6/71 (8.5\%) to $1 / 46(2.1 \%)$ over the time interval. This led to an estimated savings of $\$ 267,000$ and an estimated decreased of $315 \mathrm{NICU}$ bed days from lower IVH in preterm neonates.
\end{abstract}

Conclusions: We applied Six Sigma principles facilitating an interdisciplinary team approach to improve intraventricular hemorrhage in preterm neonates $\leq 32$ weeks. Through focused problem assessment, verification of process, and validation of outcomes we improved intraventricular hemorrhage rates substantially from 6 to 1 .

"What is already known on this topic?"

Delayed cord clamping may improve outcomes in preterm deliveries $\leq 32$ weeks

Six-Sigma may provide a framework for improving care by care analysis of medical processes

Preterm deliveries $\leq$ result in significant expense and morbidity to intraventricular hemorrhage (IVH)

"What this paper adds"

Delayed cord clamping improved outcomes in $\leq 32$ week deliveries

Six-Sigma allows for significant improvement in process with impact on clinical outcomes

Even small reductions in IVH $\leq 332$ weeks provide significant improvement in clinical outcome and huge healthcare savings

\section{Objectives and introduction}

The purpose of this research was to evaluate the impact of applying Six-Sigma methodology, specifically, the Define-Measure-AnalyzeImprove-Control (DMAIC) and Lean tools to decrease the rate of intraventricular Grade III and Grade IV hemorrhage in preterm neonates $\leq 32$ weeks. Our program involved introduction of delayed cord clamping of 30 seconds at delivery and care bundles in the neonatal intensive care unit to decrease IVH.

Our institution is the largest hospital in West Virginia. Our institution is a non-profit, academic, regional tertiary referral center. The hospital system performs 37,454 inpatient discharges and 530,861 out-patient visits annually. Our system is the largest provider of uncompensated care in West Virginia and the largest Medicaid provider in West Virginia. Major services include cardiology, obstetrical/ gynecologic, neurology, orthopedic, urologic, trauma, general surgical, cardiothoracic, and neonatology services. Our institution has a Level

*Correspondence to: Byron C Calhoun, 800 Pennsylvania Avenue, Charleston, WV 25302, USA, Tel: 304-388-2819; Fax: 304-388-2915; E-mail: Byron.calhoun@camc.org

Key words: six Sigma, delayed cord clamping

Received: August 07, 2018; Accepted: August 23, 2018; Published: August 27, 2018 
III Neonatal Intensive Care Unit and a Level I Trauma Center. Over the past five years, our institution has provided in excess of $\$ 160$ million for care of patients who cannot pay and our institution provides over $\$ 20$ million per year in subsidies for educational programs. The hospital system provides training for 140 medical residents.

As a provider of Level III Neonatal Intensive Care, our institution's Women's and Children's Hospital delivered 169 preterm neonates $\leq 32$ in the study year. Historically in the year prior to our study (2014), we found 6/71 (8.5\%) Grade III and Grade IV IVH in our population.

More recently Six Sigma tools have been applied to the nonmanufacturing realm, particularly service industries including health care. Lean Six Sigma has been useful in the process to improve healthcare outcomes and change institutional behaviors [1-6]. Six Sigma is particularly useful in improving health care processes when iterative tasks and closing loops are necessary, (i.e. delayed cord clamping and nursing bundles in NICU) [3,5]. Integration of statistical analysis by comparison of cohorts of data, analysis of root causes, and point-of-use observation were tools we used to improve enrollment in neonatal IVH prevention.

\section{Methods}

Four key concepts in the utilization of a structured Six-Sigma approach in a clinical teaching environment include:

1. Utilization of the "Change Acceptance Process, (CAP)." Projects to improve patient centered care processes must be the product of a unified team with a common tie that binds. This approach to readying the team for change and sustained performance improvement is best described using the formula, Quality = Acceptance $\mathrm{x}$ Effectiveness. The key variable in this equation is Acceptance.

2. Obtaining the "Voice of the Customer, (VOC)." This process focuses on getting input and feedback from not only persons providing care, i.e. attending physicians, resident physicians and nursing/clinical staff, but from all persons who are stakeholders in the need to improve upon the current level of performance. These persons will typically include team members who care for patients, delivering personnel, and certainly the patient. An effective method to gain VOC is to use (CAP) and designated Work Out, (WO) tools to define the issues, build the team and shape the vision needed for change.

3. Identifying and attempting to control variation and reduction of IVH in the provision of care. One of the most effective starting points to the reduction of variability in a clinical environment is to fully understand the demands of service provided.

4. Commitment to driving the improvement based on data. All members of the team must identify and commit to a defined set of quantifiable data points that will be utilized to monitor performance on a continuous basis.

Key to the success of any project involves designation of a project Champion. The Champion provides the essential drive leading to change in a large and complex healthcare organization which has many important priorities and finite resources. The Champion supports the project and navigates the organizational barriers as they present.

Our OB/GYN Clinical Improvement Champions consisted of the following: the Maternal-Fetal Medicine Staff, NICU charge nurse, and a staff neonatologist.

In addition to project Champions, other critical success factors consisted of alignment between operational administration and academic faculty expert, and a detailed and updated action and communication plan.

Access and clinical efficiency improvement in NICU and L\&D was managed using the well tested Six Sigma approach of Define-MeasureAnalyze-Improve-Control, (DMAIC). Each stage of the project is managed according to a structured process that can be outlined as follows:

\section{Define: Who are the customers and what are their priorities?}

Measure: How is the process performing and how is it measured?

Analyze: What are the most important causes of the defects?

Improve: How do we remove the causes of the defects?

Control: How can we maintain the improvements?

\section{Define}

Critical to the process was the deployment of the DMAIC principles. First we sought to Define. (Figure 1) The team utilized several interdisciplinary WO sessions to define who the customers are and to gather VOC. The tools for collecting VOC consisted of: interdisciplinary collaborative sessions, staff WO sessions including representatives from all internal customer segments, patient satisfaction surveys, and safety and grievance reports.

We identified our customers as: internal to the organizationstaff, residents, attending physicians, nursing, and external to the organization- patients and their families. The Patient VOC showed significant distress, prolonged hospitalization, and increased expense to families as result of IVH. The Residents'/Staff VOC listed unstructured approach to delayed cord clamping, lack of knowledge of benefit to neonates, and attending practice variance. Nursing personnel found the variance in the care bundles and specific guidelines for prevention of IVH in the preterm neonates frustrating and difficult to follow. The key points from the Define Stage/ VOC process showed the patients were not getting the best possible care and the Residents/Staff/Nursing were frustrated with the significant rate of IVH in the preterm neonates.

The next step in the Define stage was to clearly identify what were the Critical to Quality, (CTQ), factors in the project. This was accomplished by continuing with the CAP/WO process throughout the Define stage. CTQ identification is important in order to ensure the team is unified and focused the "Critical X's".

What are the external customer CTQ's? (Patients/Families)?

- We want a healthy preterm neonate.

- We want the best practices for our neonate.

- We want the care our neonate needs.

What are the internal customer CTQ's? (Residents/Staff/Nursing)?

- We want delayed cord clamping defined ( $\leq 32$ weeks gestation for 30 seconds)

- We want bundles of care in the NICU that are consistent and easy to follow

- We want to decrease our number of IVH by $50 \%$ over the next year

Paramount to the CAP/WO process in the teaching hospital environment was to clearly establish a clinical environment within a business model that is viable and economically sustainable. 


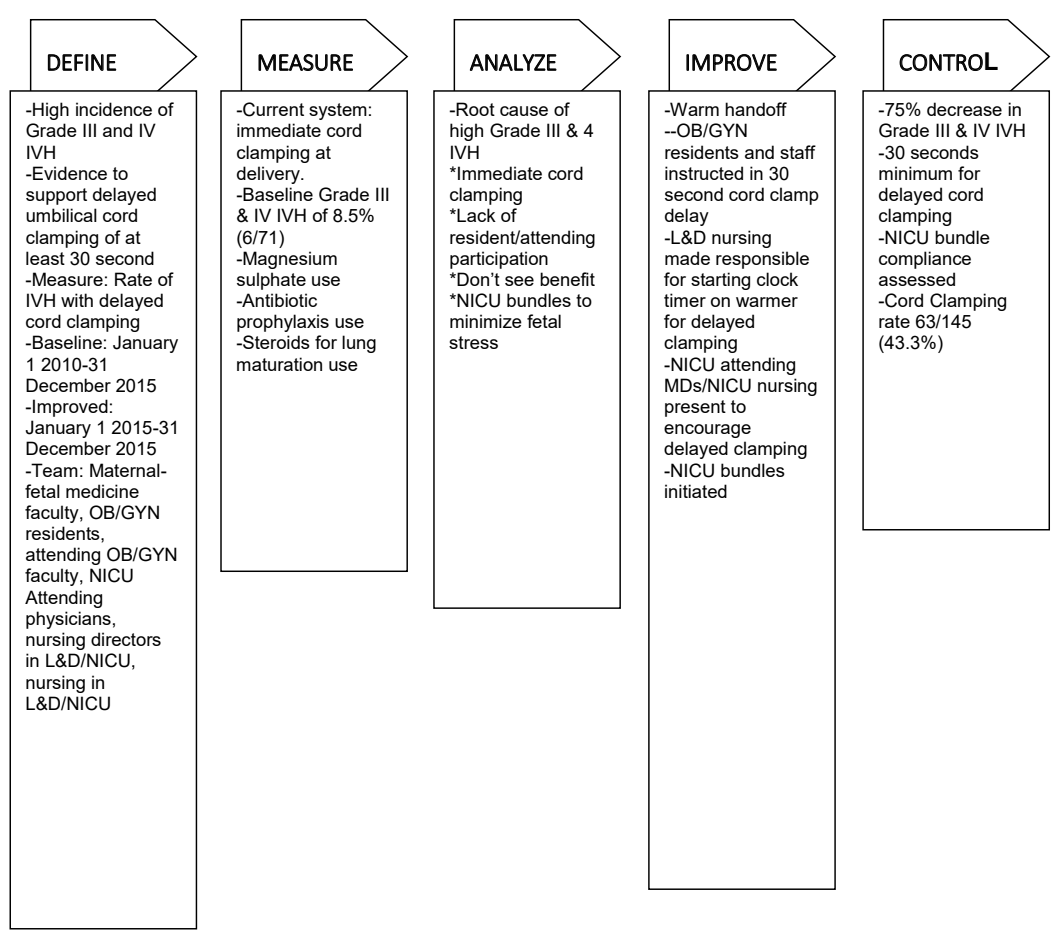

Figure 1. Lean Six Sigma DMAIC to improve delayed cord clamping

What was the project business case of improving the rate of IVH? Estimates based on decreased rates of IVH put the direct economic impact to be $\$ 267,750$ ( $\$ 850 /$ day NICU costs X reduction 5 neonates X 63 days average for Grad III or IV IVH) annually for decreased hospitalizations alone in NICU. Other significant opportunities were present for "systems based learning" as well as "clinical based learning" of the residents, faculty, nursing, improved neonatal outcomes, and protection of State disproportionate share payment to the Hospital.

What was the critical X's to develop a system that promotes decreased rates of IVH and reliability of care? The critical aspects were $\mathrm{X} 1$ = Delayed Cord clamping for 30 seconds, X2 = NICU Bundles (Table 1), X3 = Attending Physician Supervision, X4 = Pre-delivery steroids (when applicable), X5 = Pre-delivery magnesium sulphate (when applicable) and X6 = Actionable information on hand.

\section{Measure}

Next in the DMAIC process we needed to measure. (Figure 2) This meant setting performance standards. The current state of performance revealed: 6/71 IVHs per year (2013) prior to initiation of delayed cord clamping and NICU bundles (Table 1).

We needed to find the right $\mathrm{Y}$ (CTQ) to Measure. How will it be measured? We chose the following Y's: $Y=$ Number of Grade III and IV IVHs, $\mathrm{Y}=$ adherence to 30 second delayed cord clamping, and $\mathrm{Y}=$ adherence to NICU care bundles. (Figure 3-"Fishbone")

Our defects would be either, not providing a 30 second delayed cord clamping, and, non-adherence to the NICU care bundles. A structured approach was put in place to repeatedly collect and measure data in a uniform and reproducible manner.

\section{Analyze}

Once the measures were identified and agreed upon from a standpoint of importance, analysis of data became paramount. (Figure
Table 1. IVH reduction nursing bundles

\begin{tabular}{|c|c|c|}
\hline \multicolumn{3}{|c|}{$\begin{array}{l}\text { IVH Reduction Nursing Bundles } \\
<1500 \mathrm{~g} \text { or }<32 \text { weeks }\end{array}$} \\
\hline \multicolumn{3}{|c|}{ Day 1} \\
\hline Did not receive an IV Bolus & Yes & No \\
\hline Maintain temperature (36.5-37.5) & Yes & No \\
\hline Minimal handling (guidelines revised) & Yes & No \\
\hline Head midline position & Yes & No \\
\hline Head of the bed elevated ( 30 degrees) & Yes & No \\
\hline Suctioning PRN & Yes & No \\
\hline No cuff pressure & Yes & No \\
\hline Did not receive Sodium Bicarbonate & Yes & No \\
\hline \multicolumn{3}{|c|}{ Day 2} \\
\hline Did not receive an IV Bolus & Yes & No \\
\hline Maintain temperature (36.5-37.5) & Yes & No \\
\hline Minimal handling (guidelines revised) & Yes & No \\
\hline Head midline position & Yes & No \\
\hline Head of the bed elevated ( 30 degrees) & Yes & No \\
\hline Suctioning PRN & Yes & No \\
\hline No cuff pressure & Yes & No \\
\hline Did not receive Sodium Bicarbonate & Yes & No \\
\hline \multicolumn{3}{|c|}{ Day 3} \\
\hline Did not receive an IV Bolus & Yes & No \\
\hline Maintain temperature (36.5-37.5) & Yes & No \\
\hline Minimal handling (guidelines revised) & Yes & No \\
\hline Head midline position & Yes & No \\
\hline Head of the bed elevated ( 30 degrees) & Yes & No \\
\hline Suctioning PRN & Yes & No \\
\hline No cuff pressure & Yes & No \\
\hline Did not receive Sodium Bicarbonate & Yes & No \\
\hline
\end{tabular}

4) Examination of the data revealed several sources of variation that led to adverse clinical outcomes. The team began to implement repeated cycles of "tests of change" focused on steps in the throughput process that cumulatively resulted in quantifiable changes in the way work 


\section{MEASURE}

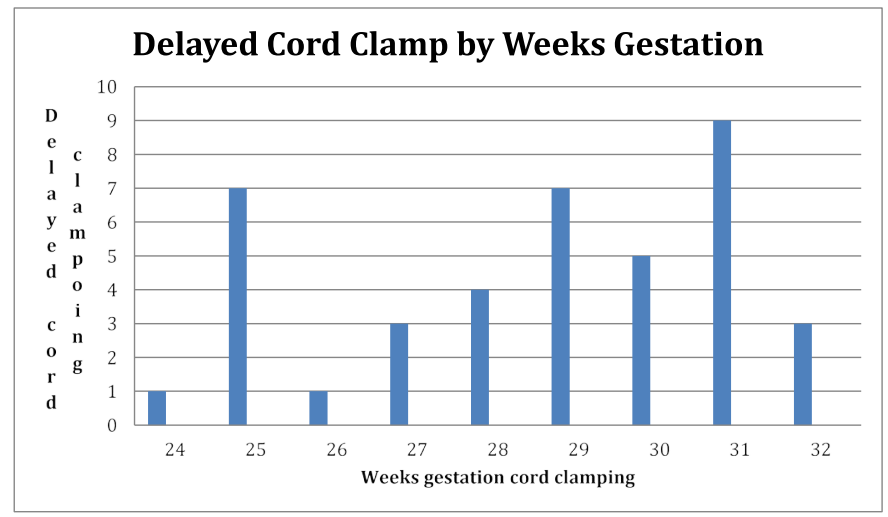

Figure 2. Cord clamping by weeks gestation

Charleston Area Medical Center Labor and Delivery

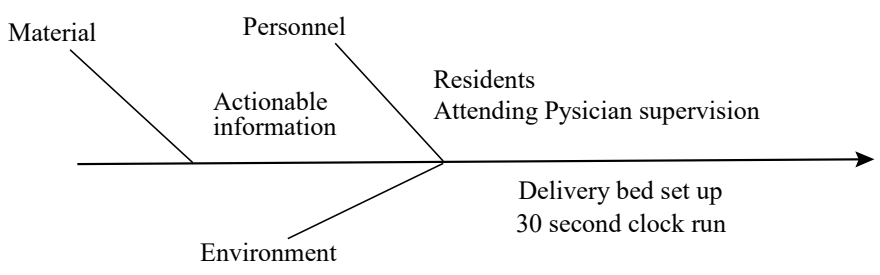

Figure 3. Fishbone of variables on $\mathrm{X}$ and $\mathrm{Y}$ axes

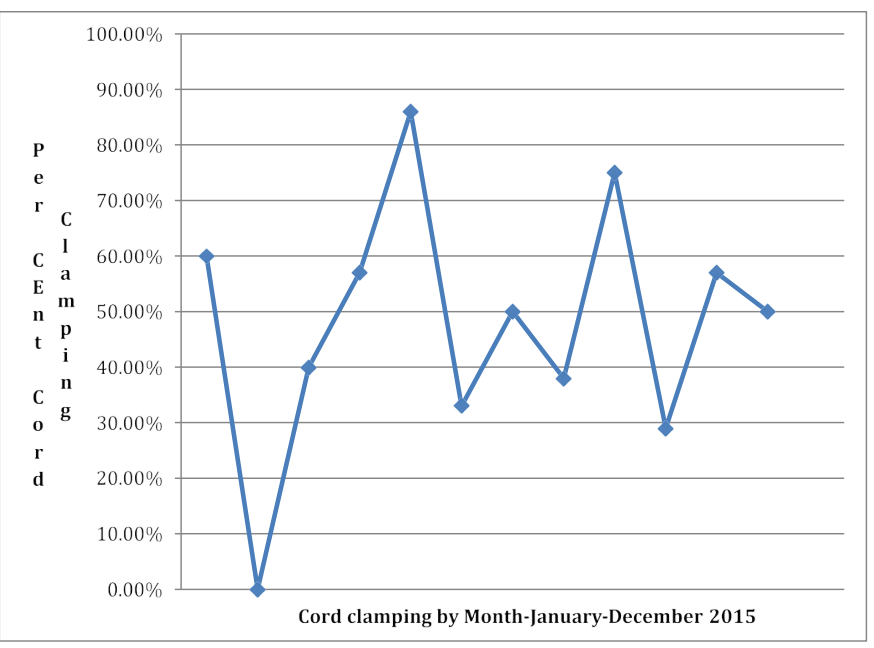

Figure 4. Control chart: 30 second cord clamp by month (January-December 2015)

was organized and completed. Change occurred in several areas. These changes were driven by the team with data in hand and the assurance that the monitoring tools were in place and operational on a go forward basis.

\section{Improve}

The improvements (from DMAIC) followed from the changes implemented. An overview of our changes included:

- Resident, attending, L\&D nursing, and NICU personnel education on why and how to perform the delayed cord clamping for 30 seconds using the Apgar timer on the resuscitation bed.

- Standard order sets for preterm delivery patients reviewed for use of steroids and magnesium sulfate
- NICU neonatal care bundles standardized to provide minimal neonatal stimulation

- Periodic assessment of delayed cord clamping by residents/staff

- Through application of the CAP process, a cultural change occurred in which the staff, residents and attending physicians committed to holding each other accountable for delayed cord clamping, information readiness and communication.

\section{Control}

In an environment that is historically variable, ongoing tests of change and repeat cycles of learning are now beginning to demonstrate elements of improved "control." By no means do we have the type of sigma scores represented in the automobile manufacturing or air travel industry but we do have the capacity and knowledge to now use control charts to monitor performance for improvement of variation.

\section{Results}

As a consequence of the implementation of the DMAIC process, changes with delayed cord clamping and neonatal care bundles were made. The total number of IVHs decreased from 6/71 IVHs per year (2014) prior to initiation of delayed cord clamping and NICU bundles to $1 / 42$ IVHs in the new process. Using the average number of days of stay of 63 days for a Grade III and IV hemorrhages at our institution, and, a cost of $\$ 850 /$ day, we had a decrease in hospital NICU costs for IVH with delayed cord clamping of $\$ 267,750$ [ $\$ 321,300$ (6X \$850X63) $\$ 53,550$ (1X63X \$850)] and saved 315 NICU bed days. These cost savings do not include the substantial costs for the substantial morbidity with Grade III and IV due to increased rates of CP, learning disabilities, blindness, and deafness.

Over the one-year period compared, delayed cord clamping $\leq 32$ weeks went from $0 \%$ to $47 / 96$ (49\%). Of the 49 patients who did not undergo delayed cord clamping, we found it was due to multiparity, chorioamnionitis, fetal sepsis, and/or attending choice.

Areas targeted by our Six-Sigma process for continued improvement include: increasing number of neonates born $\leq 32$ weeks who underwent cord clamping, increasing number of neonates who had proper NICU bundle care, continuing emphasis on pre-delivery steroids, magnesium sulfate and prophylactic antibiotics. (See Table 1 for Bundles)

\section{Conclusions}

Application of the Six-Sigma management principles improved neonatal Grade III and IV IVH rates. Focused problem assessment, verification of process, and validation of outcomes, anchor any attempts to couple change with decreased Grade III and IV IVH. Less Grade III and IV IVH results in less NICU hospital time, significant decrease in morbidity (i.e. seizures, $\mathrm{CP}$, and learning disability), and significant cost savings (over $\$ 267,000$ in 1 year in NICU costs alone and 315 bed days in the NICU). In summary, our project supported the use of simple delayed cord clamping of just 30 seconds with neonatal nursing bundles in neonates $\leq 32$ weeks to significantly decrease Grade III and Grade IV hemorrhage rates.

\section{Directions for future research}

Future research in our clinic will include continued tracking of our processes to validate our present success and IVH. We also anticipate applying the concept of embedded personnel workflow to our other processes to provide on-site care, continued educational efforts, and standardization of delivery for neonates $\leq 32$ weeks. All of these areas 
are amenable to embedded personnel in the workflow and ought to yield increased enrollment and compliance.

\section{Discussion}

Our lean process led to a clinically significant decrease in Grade III and IV IVH in neonates $\leq 32$ weeks and cost savings of $\$ 267,750$ for NICU hospital costs alone. Without formal evaluation of the process, merely initiating delayed cord clamping would not be sufficient in decreasing IVH. By no means do we have the type of sigma scores represented in automobile manufacturing or aerospace industry, but, we do have capacity and knowledge to now use control charts to monitor performance for improvement of variation.

Application of Six-Sigma management principles to residency teaching outpatient clinic resulted in a clinically significant decrease in Grade III and IV IVH (decreasing by 5 in one year) without increasing personnel or personnel costs. We have not yet reached the goal of Six Sigma of $99.9997 \%$ error free processes enrollment and work flow. Perhaps the use of a workflow engine providing an automated, measurable and improved process for tracking delayed cord clamping and NICU bundle compliance in a complex educational environment with 12 residents, multiple staff physicians, and nurse midwives could assist in the deployment of the initiative. Focused problem assessment, verification of process, and validation of outcomes anchor any attempts to couple increased use of delayed cord clamping and NICU bundles with improved patient care in the setting of a residency program. We think continued engagement, iterative evaluations, and refinement of our work flow will eventually allow us to achieve a $98+\%$ delayed cord clamping and adherence to NICU bundles in eligible neonates necessary to our system.

\section{Funding}

No funding from external sources was used in the completion of this project. The research was conducted as an exempt protocol under quality improvement.

\section{References}

1. Pande PS, Neumann RP, Cabanagh RR (2000) The Six Sigma Way: How GE, Motorola, and Other Top Companies are Honing Their Performance. MCGraw-Hill, New York, NY, USA.

2. Chassin R (2008) The Six Sigma initiative at Mount Sinai Medical Center. Mt Sinai J Med 75: 45-52. [Crossref]

3. Raab SS, Andrew-JaJa C, Condel JL, Dabbs DJ (2006) Improving Papaniaolau test quality and reducing medical errors by using Toyota production system methods. Am J Obstet Gynecol 194: 57-64. [Crossref]

4. Babensky JA, Roe J, Bolton R (2005) Lean Sigma-Will it work for Healthcare? J Healthc Inf Manag 19: 39-44. [Crossref]

5. de Koning H, Verver JPS, van den Heuvel J, Bisgaard S, Does RJ (2006) Lean six sigma in healthcare. J Healthc Qual $28: 4-11$. [Crossref]

6. Calhoun BC, Goode J, Simmons K (2011) Process improvement of pap smear tracking in a women's medicine center clinic in residency training. J Healthc Qual 33: 25-32. [Crossref]

Copyright: (C2018 Clark S. This is an open-access article distributed under the terms of the Creative Commons Attribution License, which permits unrestricted use, distribution, and reproduction in any medium, provided the original author and source are credited. 